\title{
Labdanes and Withanolides from Physalis coztomatl
}

Ana-L. Pérez-Castorena, ${ }^{* \dagger}$ Ruth F. Oropeza ${ }^{\dagger}$ Alma R. Vázquez, ${ }^{\dagger}$ Manida Martínez ${ }^{\ddagger}$, Emma Maldonado ${ }^{\dagger}$

Instituto de Química, Universidad Nacional Autónoma de México, Circuito Exterior, Ciudad Universitaria, Coyoacán 04510, D.F., México

Facultad de Ciencias Naturales, Universidad Autónoma de Querétaro, Cerro de las Campanas s. n., Querétaro, México.

${ }^{1} \mathrm{H}$ NMR spectrum of physacoztomatin (1)

${ }^{1} \mathrm{H}$ NMR spectrum of physacoztolide A (4)

${ }^{1} \mathrm{H}$ NMR spectrum of physacoztolide B (5)

${ }^{1} \mathrm{H}$ NMR spectrum of physacoztolide C (6)

${ }^{1} \mathrm{H}$ NMR spectrum of physacoztolide D (7)

${ }^{1} \mathrm{H}$ NMR spectrum of physacoztolide E $(\mathbf{8})$

\footnotetext{
${ }^{*}$ To whom correspondence should be addressed. Tel. (5255) 56-224412. Fax (5255) 56-162217. E-mail alperezc@servidor.unam.mx.

† Instituto de Química, UNAM, contribution No. 0000.

${ }^{\star}$ Facultad de Ciencias Naturales, UAQ
} 